\title{
Compact third-order limiter functions for finite volume methods
}

\section{Doctoral Thesis}

Author(s):

Čada, Miroslav

Publication date:

2009

Permanent link:

https://doi.org/10.3929/ethz-a-005907275

Rights / license:

In Copyright - Non-Commercial Use Permitted 
Diss. ETH No. 18346

\title{
Compact Third-Order Limiter Functions for Finite Volume Methods
}

\author{
A dissertation submitted to the
}

ETH ZURICH

for the degree of

Doctor of Sciences

$$
\begin{aligned}
& \text { presented by } \\
& \text { MIROSLAV ČADA }
\end{aligned}
$$

Dipl. Geo Physiker, Ludwig-Maximilians University, Munich

born January 14, 1976

citizen of Slovak Republic

accepted on the recommendation of

Prof. Dr. Manuel Torrilhon, examiner

Prof. Dr. Rolf Jeltsch, co-examiner 


\section{Abstract}

In this thesis we consider finite volume methods for the numerical solution of conservation laws modeled by systems of nonlinear hyperbolic partial differential equations. We are in particular interested in highorder accurate numerical approximations of conservation laws.

Numerical approximations of nonlinear hyperbolic partial differential equation are particularly challenging, since their solution develops jump discontinuities in finite time. In order to achieve high-order accurate numerical approximation to nonlinear smooth functions, we introduce new high-order accurate reconstruction functions for the spatial approximation of hyperbolic partial differential equations. We therefore employ and generalize the idea of non-oscillatory double-logarithmic reconstruction of Artebrant and Schroll [5]. The proposed spatial approximation is based upon a new class of limiter functions, which recover efficiently and with high-order accuracy smooth, as well as discontinuous functions.

The result is a an efficient third-order accurate scheme with a compact three point stencil. The interface values between two neighboring cells are obtained by a single nonlinear limiter function. In contrast to classical limiter functions, the new limiters handle discontinuities as well as local extrema within the standard semi-discrete TVD-MUSCL [77] framework utilizing an explicit SSP Runge-Kutta time marching scheme [18]. The shape preserving properties of the reconstruction are significantly improved, resulting in sharp, accurate and symmetric shock capturing.

An extensive analysis of existing limiters, reveals the major drawbacks associated with second-order accurate TVD reconstructions. Smearing of discontinuities, clipping and squaring effects of smooth extrema associated with classical second-order TVD limiters are examined. Stability analysis confirms, that squaring effects, originally associated with compressive limiter are essentially local instabilities, which trigger exponential growth of certain wave modes. The proposed new class of third-order accurate limiter completely avoids these defects, producing perfectly symmetric results.

Although the new algorithm employs a three-stage, third-order accurate time marching scheme, its computational efficiency is enhanced due to large allowable Courant number $\nu$. As indicated by the von Neumann stability analysis, large time steps, satisfying $\nu \lesssim 1.6$ are admissible and in some cases even preferable. Utilizing third-order time integration, the dissipation of high frequency waves gets reduced

with large Courant numbers $1.0 \leq \nu \lesssim 1$.6. Furthermore third-order accurate schemes get unstable for 
high frequency waves for $\nu \lesssim 1.6$, but remain stable for small frequencies. In contrast to second-order accurate schemes, where basically all Fourier modes get unstable, resulting eventually in wave dispersion.

To test the proposed scheme and to verify the theoretical analysis, we conducted a series of numerical experiments for a variety of hyperbolic partial differential equations. We calculated numerical approximation for the linear advection equation, the nonlinear Burger's equation, the nonlinear Buckley-Leverett equation, the Euler equation (in $1 \mathrm{~d}$ and $2 \mathrm{~d}$ ) of ideal gas dynamics and the ideal magneto-hydrodynamicdynamic equations. Furthermore we also considered balance equations with stiff relaxation sources. We tested the scheme on the shallow water equations, the Broadwell system of kinetic gas theory and Grad's 13 moment system of extended thermodynamics of rarefied gas dynamics.

The proposed scheme was compared to several well established high-order accurate methods, such as Artebrant and Schroll's LDLR [5], Marquina's LHHR [42], ENO / WENO [60] and several TVD schemes. The results indicate the superiority of the proposed limiter over classical second-order TVD limiters and their modifications. The new scheme also compares favorably with third-order methods such as LDLR, LHHR and ENO / WENO. A major part of the results of this work can be found in condensed form in $[78-80]$. 


\section{Zusammenfassung}

In dieser Arbeit betrachten wir Finite Volumen Verfahren für die numerische Lösung von Erhaltungsgleichungen. Erhaltungsgleichungen werden durch Systeme von nichtlinearen hyperbolischen partiellen Differentialgleichungen modelliert. Wir sind insbesondere an Verfahren interessiert, die Erhaltungsgleichungen mit hoher Genauigkeit approximieren.

Numerische Approximationen nichtlinearer Erhaltungsgleichungen sind eine besondere Herausforderung, da ihre L̈̈ung Unstetigkeiten in endlicher Zeit entwickelt. Um Lösungen von hoher Genauigkeit zu erreichen, führen wir eine neue Klasse von Rekonstruktionsfunktionen für die räumliche Approximation von hyperbolischen Differentialgleichungen ein. Zu diesem Zweck verallgemeinern wir die Idee von "Oszillations-freien, doppelt-logarithmischen" Rekonstruktionsfunktionen von Artebrant und Schroll [5]. Die hier vorgeschlagene räumliche Rekonstruktion basiert auf einer neuen Klasse von Limiter-Funktionen, die effizient und mit hoher Genauigkeit sowohl glatte, als auch diskontinuierliche Funktionen approximieren.

Als Ergebnis erhalten wir ein kompaktes Finite Volumen Verfahren, bei welchem Zwischenzellwerte mit dritter Ordnung Genauigkeit approximiert werden. Kompakte Verfahren benützen lediglich direktbenachbarte Zellmittelwerte für die Rekonstruktion von Zwischenzellwerten. Die Zwischenzellwerte werden mit Hilfe einer einzigen nichtlinearen Limiter-Funktion berechnet. Im Gegensatz zu klassischen Limiter-Funktionen, approximieren die neuen Limiter sowohl Unstetigkeiten, als auch glatte Extrema im Rahmen der bekannten semi-diskreten TVD-MUSCL Verfahren [77]. Für die Zeitintegration wenden wir explizite SSP Runge-Kutta Verfahren [18] an. Die Anwendung der neuen Limiter verbessert signifikant die Qualität der numerischen Approximation von Unstetigkeiten.

Eine umfassende Analyse von existierenden Limitern, zeigt die Nachteile der dazugehörigen Finite Volumen Verfahren zweiter Ordnung. Effekte, wie das Ausschmieren von Unstetigkeiten, das Abschneiden und Komprimieren von glatten Extrema, welche bei der Anwendung von Verfahren zweiter Ordnung auftreten, werden untersucht. Mithilfe von Stabilitäts-Analysen können wir zeigen, dass die Kompression von glatten Funktionen im Wesentlichen auf lokale Instabilitäten zurc̈kzuführen ist. Die eingeführten Verfahren von dritter Ordung Genauigkeit beheben diese Mn̈gel vollständig.

Obwohl wir für die Zeitintegration ein drei stufiges Runge-Kutta Verfahren verwenden, wird die Kon- 
vergenzgeschwindigkeit des Verfahrens durch grosse Courant-Zahlen $\nu$ verbessert. Die von NeumannStabilitẗs-Analyse deutet an, dass grosse Zeitschritte $\nu \lesssim 1.6$ zulässig und in einigen Fällen sogar wünschenswert sind. Bei der Verwendung von Integrationsverfahren mit dritter Ordnung Genauigkeit im der Zeit, wird die Dissipation von hochfrequenten Wellen bei grossen Courant-Zahlen $1 \leq \nu \lesssim 1.6$ reduziert. Im Gegensatz zu Verfahren zweiter Ordnung räumliche und zeitlicher Genauigkeit, sind Verfahren dritter Ordnung dissipativ. Das führt bei Instabilität, $\nu>1.6$, zur Verstärkung von hochfrequenten, aber nicht niederfrequenten Wellen.

Um unser Verfahren und die theoretischen Ergebnisse zu validieren, haben wir eine Reihe von numerischen Experimenten für verschiedene Erhaltungsgleichungen durchgeführt. Dazu gehören, lineare Advektions-Gleichung, die nichtlineare Burger-Gleichung, die nichtlineare Buckley-Leverett Gleichung, die Euler Gleichungen der idealen Gasdynamic (in 1d und 2d) und das System der idealen Magnetohydrodynamik. Desweiteren wurde steife Relaxationssysteme berücksichtigt. Wir haben unser Verfahren am Beispiel der Seichtwasser-Gleichungen, des Broadwell-Systems und Grad 13 Momenten-Systems getestet.

Das eingeführte Verfahren wurde mit verschiedenen hoch auflösenden Methoden verglichen. Dazu gehören Artebrant and Schroll's LDLR [5], Marquina's LHHR [42], ENO / WENO [60] und verschiedenste TVD Verfahren. Die Ergebnisse zeigen die Überlegenheit der vorgeschlagenen Limiter-Funktionen ber klassische TVD Limiter zweiter Ordnung. Das neue Verfahren bewährt sich auch im Vergleich mit hoch auflösenden Methoden, wie LDLR, LHHR und ENO / WENO. Ein Grossteil der in dieser Arbeit vorgestellten Ergebnisse ist in kondensierter Form in den Artikeln [78-80] nachzulesen. 